\title{
Clinical Trial on the Characteristics of Zheng Classification of Pulmonary Diseases Based on Infrared Thermal Imaging Technology
}

\author{
Jin-xia Ni, ${ }^{1}$ Si-hua Gao, ${ }^{2}$ Yu-hang Li, ${ }^{2}$ Shi-lei Ma,, Tian Tian, ${ }^{2}$ Fang-fang Mo, \\ Liu-qing Wang, ${ }^{3}$ and Wen-zeng $\mathrm{Zhu}^{4}$ \\ ${ }^{1}$ Dongzhimen Hospital Affiliated to Beijing University of Chinese Medicine, Beijing 100700, China \\ ${ }^{2}$ Beijing University of Chinese Medicine, Beijing 100029, China \\ ${ }^{3}$ China Academy of Chinese Medicine Sciences, Beijing 100029, China \\ ${ }^{4}$ Guang'anmen Hospital, China Academy of Chinese Medicine Sciences, Beijing 100053, China \\ Correspondence should be addressed to Si-hua Gao; gaosihua1216@163.com
}

Received 7 January 2013; Revised 3 March 2013; Accepted 14 March 2013

Academic Editor: Zhaoxiang Bian

Copyright (C) 2013 Jin-xia Ni et al. This is an open access article distributed under the Creative Commons Attribution License, which permits unrestricted use, distribution, and reproduction in any medium, provided the original work is properly cited.

\begin{abstract}
Zheng classification study based on infrared thermal imaging technology has not been reported before. To detect the relative temperature of viscera and bowels of different syndromes patients with pulmonary disease and to summarize the characteristics of different Zheng classifications, the infrared thermal imaging technology was used in the clinical trial. The results showed that the infrared thermal images characteristics of different Zheng classifications of pulmonary disease were distinctly different. The influence on viscera and bowels was deeper in phlegm-heat obstructing lung syndrome group than in cold-phlegm obstructing lung syndrome group. It is helpful to diagnose Zheng classification and to improve the diagnosis rate by analyzing the infrared thermal images of patients. The application of infrared thermal imaging technology provided objective measures for medical diagnosis and treatment in the field of Zheng studies and provided a new methodology for Zheng classification.
\end{abstract}

\section{Introduction}

Infrared thermal imaging technology is a noninvasive imaging procedure used to record the thermal patterns using infrared camera. It is widely applied in the field of biomedicine. It is believed that infrared thermal imaging cameras can potentially be used to detect subjects with fever, the cardinal symptom of SARS, and avian influenza [1]. And it has been previously utilized in the detection of breast cancer [2], in the diagnosis and management of erectile dysfunction [3], and in the evaluation of tear evaporation from ocular surface $[4,5]$. Regarded as a speedy, comprehensive, convenient, and fairly accurate means, the use of infrared thermal imaging technology offers great opportunities for the study of Zheng classification of pulmonary diseases. While the relative temperature of viscera and bowels is detected by infrared camera, the function of viscera and bowels is visualized. Previous study showed the temperature of viscera and bowels regulated in a special rule under physiological conditions [6]. Would the relative temperature of viscera and bowels of patients with pulmonary diseases from different Zheng groups change while compared with healthy people? How about the characteristics of Zheng classification of pulmonary diseases based on infrared thermal imaging technology? With these questions, we carried out these relative clinical trials.

\section{Methods}

2.1. Selection of Study Participants. 72 healthy volunteers (group A) were enrolled from community resident in Dongcheng District of Beijing. 111 patients with pulmonary disease including chronic obstructive pulmonary disease, bronchial asthma, and chronic bronchitis were derived from the outpatient of Dongzhimen Hospital Affiliated to Beijing University of Chinese Medicine. Among these patients, 
TABLE 1: The distribution of gender in different groups $(n)$.

\begin{tabular}{lccccc}
\hline Groups & $n$ & Male & Female & $\chi^{2}$ & $P$ \\
\hline Group A & 72 & 18 & 54 & & \\
Group B & 35 & 10 & 25 & 4.486 & 0.106 \\
Group C & 76 & 31 & 45 & & \\
\hline
\end{tabular}

TABLE 2: The distribution of age range in different groups $(n)$.

\begin{tabular}{lccccc}
\hline Groups & $n$ & $46 \sim 60$ years old & $61-75$ years old & $\chi^{2}$ & $P$ \\
\hline Group A & 72 & 53 & 19 & & \\
Group B & 35 & 19 & 16 & 4.728 & 0.094 \\
Group C & 76 & 46 & 30 & & \\
\hline
\end{tabular}

35 cases were diagnosed cold-phlegm obstructing lung syndrome (Group B) and 76 cases were diagnosed phlegmheat obstructing the lung syndrome (Group C). The clinical trial was approved by the Ethics Committee of Dongzhimen Hospital Affiliated to Beijing University of Chinese Medicine. The gender and age distribution was shown in Tables 1 and 2 .

2.2. Diagnosis Criteria of Chronic Obstructive Pulmonary Disease. Reference to the practical internal medicine and chronic obstructive pulmonary disease treatment guidelines (2007 revision) was written by chronic obstructive pulmonary disease study group of Chinese Society of Respiratory Diseases [7].

2.3. Diagnosis Criteria of Bronchial Asthma. Reference to the Bronchial asthma prevention and treatment guidelines was revised by Asthma study group of Chinese Society of Respiratory Diseases on November 7, 2002 [8].

2.4. Diagnosis Criteria of Chronic Bronchitis. Reference to the Chronic bronchitis clinical diagnosis and the efficacy criteria was revised by National Clinical Professional meeting of chronic bronchitis in 1979 [9].

2.5. Diagnosis Criteria of Zheng Classification of Pulmonary Diseases. Reference to the Chinese internal medicine and TCM Zheng Diagnosis and efficacy standard [10] and TCM terms [11].

2.6. Diagnosis Criteria of Phlegm-Heat Obstructing Lung Syndrome. Primary symptoms include (1) cough or (and) dyspnea; (2) yellow sticky sputum.

Associated symptoms include (1) vexation, (2) abdominal distention and fullness, (3) dry mouth and thirst and like drinking cold, (4) fever but without aversion to cold, (5) yellow urine, (6) dyschezia, (7) red tongue, (8) yellow or yellow with greasy tongue fur, and (9) slippery and rapid pulse.

2.7. Diagnosis Criteria of Cold-Phlegm Obstructing Lung Syndrome. Primary symptoms: (1) cough or (and) dyspnea (2) white sputum.
Associated symptoms include (1) oppression in the chest, (2) fear of cold and cold extremities, (3) pale tongue, (4) white with lubricating tongue fur, (5) string-like with tight pulse.

Patient with primary symptoms and two associated symptoms can be diagnosed.

\subsection{Selection Criteria to Patients with Pulmonary Diseases}

(1) In line with the diagnosis criteria of chronic obstructive pulmonary disease or bronchial asthma or chronic bronchitis.

(2) In line with the diagnosis criteria of phlegm-heat obstructing the lung syndrome or cold-phlegm obstructing lung syndrome.

(3) 46 to 75 years old.

(4) Without skin diseases or skin allergies or skin surface damage.

(5) Patients in stable condition can coordinate with the researchers to completed the trial independently.

(6) Signed informed consent.

2.9. Method of Infrared Thermal Imaging Detection. ATIRM301 medical infrared thermal imager (produced by Chongqing Wei-Lian company with $0.05^{\circ} \mathrm{C}$ temperature resolution and $3 \mathrm{mrad}$ spatial resolution) was used in this clinical trial. The scanning room temperature was controlled at $24 \pm$ $2^{\circ} \mathrm{C}$ and the humidity was $65 \%-70 \%$. In order to make the surface temperature balanced, the healthy volunteer or patients exposed completely the body and stood quietly in the scanning room for 15 minutes to adapt to the environmental temperature before being checked. The anteroposterior images were scanned and saved to be analyzed. Medical thermography analysis software was used to detect and analyze the relative temperature of viscera and bowels of healthy people and patients with different Zheng of pulmonary diseases. The relative temperature was measured in the region that viscera and bowels project.

2.10. Statistical Analysis. $\chi^{2}$ test was used to analyze the enumeration data. One-way ANOVA was used to analyze the data between the normal and different Zheng classification groups. $q$ test and LSD method were used for the paired comparisons, with $P<0.05$ denoted as significant.

\section{Results}

3.1. The Relative Temperature Characteristics of Viscera and Bowels in Different Groups. The trial showed that in the normal group and in the cold-phlegm obstructing lung syndrome group, as well as in the phlegm-heat obstructing lung syndrome group, the maximum temperature of viscera and bowels was taken in the lung region, followed by the lower temperature in both the descending colon and the ascending colon regions; see Figures 1, 2, and 3. 


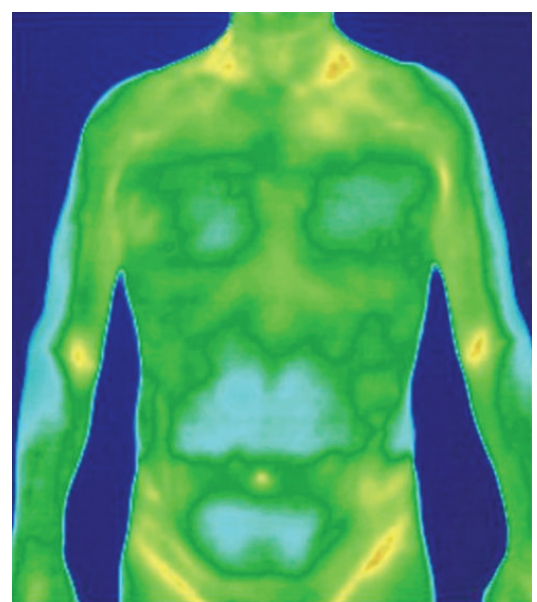

FIGURE 1: The infrared thermal images of healthy people.

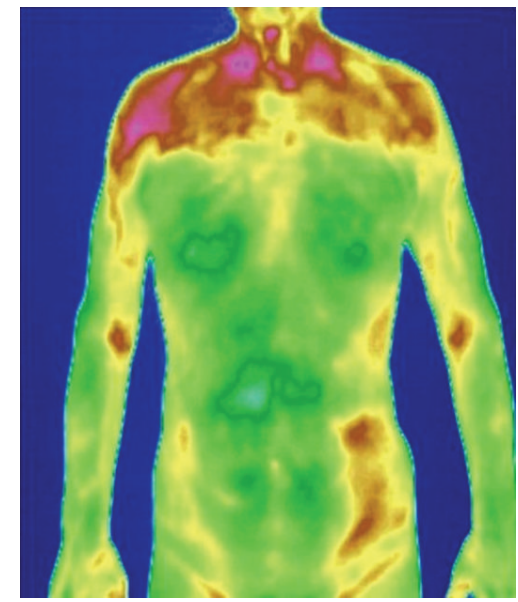

FIGURE 2: The infrared thermal images of patient with phlegm-heat obstructing lung syndrome.

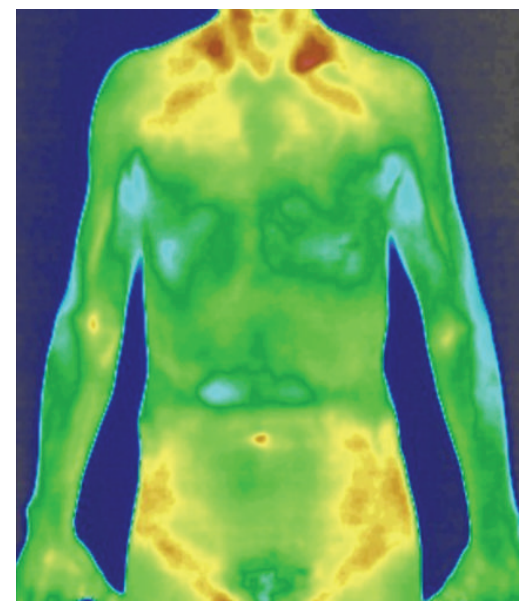

FIGURE 3: The infrared thermal images of patient with cold-phlegm obstructing lung syndrome.
TABLE 3: The temperature of ascending colon, descending colon, and small intestine compared with different groups $\left({ }^{\circ} \mathrm{C} ; \bar{X} \pm s\right)$.

\begin{tabular}{|c|c|c|c|c|}
\hline Groups & $n$ & Ascending colon & Descending colon & Small intestine \\
\hline Group A & 72 & $31.09 \pm 0.98$ & $31.12 \pm 0.92$ & $30.26 \pm 0.89$ \\
\hline Group B & 35 & $31.47 \pm 1.08$ & $31.60 \pm 1.08^{\Delta}$ & $30.60 \pm 1.03$ \\
\hline Group C & 76 & $31.77 \pm 1.03^{\Delta}$ & $31.92 \pm 1.00^{\boldsymbol{\Lambda}}$ & $31.03 \pm 1.08^{\mathbf{4}}$ \\
\hline
\end{tabular}

TABLE 4: The temperature of stomach, lung, liver and kidney compared with different groups $\left({ }^{\circ} \mathrm{C} ; \bar{X} \pm s\right)$.

\begin{tabular}{|c|c|c|c|c|c|}
\hline Groups & $n$ & Stomach & Lung & Liver & Kidney \\
\hline $\begin{array}{l}\text { Group } \\
\text { A }\end{array}$ & 72 & $30.34 \pm 1.19$ & $31.33 \pm 0.98$ & $30.63 \pm 1.09$ & $30.30 \pm 1.04$ \\
\hline $\begin{array}{l}\text { group } \\
\text { B }\end{array}$ & 35 & $30.47 \pm 1.16$ & $31.73 \pm 1.00^{\triangle}$ & $30.88 \pm 1.15$ & $30.49 \pm 0.97$ \\
\hline $\begin{array}{l}\text { group } \\
\text { C }\end{array}$ & 76 & $30.94 \pm 1.21^{\mathbf{\Lambda}}$ & $32.18 \pm 0.95^{\mathbf{\Lambda}}$ & $30.98 \pm 1.10$ & $30.62 \pm 1.02$ \\
\hline
\end{tabular}

3.2. The Comparison of Relative Temperature of Viscera and Bowels among Different Groups. The results showed that the temperature characteristics of viscera and bowels were different in different Zheng classifications of pulmonary diseases. Compared with normal group, the relative temperature of lung, descending colon, ascending colon, small intestine, and stomach significantly increased in the phlegm-heat obstructing lung syndrome group, and there was a statistical difference. While compared with normal group, the relative temperature of lung and descending colon was significantly increased in the cold-phlegm obstructing lung syndrome group, and there was a statistical difference, but the relative temperature of ascending colon, small intestine, and stomach was not significantly different. Compared with cold-phlegm obstructing lung syndrome group, the relative temperature of lung and small intestine significantly increased in phlegmheat obstructing lung syndrome group, and there was a statistical difference. See Tables 3 and 4.

\section{Discussion}

Syndrome differentiation is one of the important guidance and principles in the clinic of traditional Chinese medicine. Zheng classification is the basis of syndrome differentiation, and it is a method to distinguish between the health and disease by correlation of all four examinations. The research on Zheng classification is one of the cut-in points on integration of traditional Chinese and Western medicine [12]. It is an important way to classify diseases with syndrome differentiation and a mutual supplement for integration of traditional Chinese and Western medicine, also an important measure and approach for developing Chinese medicine and innovating biomedicine. It is meaningful in both theoretical guidance and clinical practice [13]. 
Epidemiological studies have shown that the main leading causes of death in China urban population in 20022003 were cancer and heart diseases, respectively; the death due to respiratory diseases accounted for $15.63 \%$ of the total mortality year round. In 2006, the death due to respiratory diseases was in the fourth place [14]. The four leading causes of death in all scenarios were projected to be ischaemic heart disease, cerebrovascular disease (stroke), HIV/AIDS, and COPD [15]. Thereby, the research on Zheng classification of pulmonary diseases is of great significance in guiding clinical practice. Although in the 1980s a TCM syndromes standard was studied, but the name of Zheng, the Zheng classification and the diagnosis of Zheng are not yet unified [16]. It is practical that the disease classification should be a reference frame and the research on Zheng classification should be carried out on the basis of the disease classification [12]. In this clinical trial, the common pulmonary diseases including chronic obstructive pulmonary disease, bronchial asthma, and chronic bronchitis were referenced as frame and the research on Zheng classification was studied.

Compared with previous Zheng classification study, the infrared thermal imaging technology was used in this trial. The two opposing Zheng classification including cold and heat of pulmonary disease were studied to distinguish the characteristics by infrared thermal imaging.

Infrared thermal imaging technology is a functional imaging technique. The body temperature can be continuously traced objectively and accurately by medical infrared imaging at any point and two-dimensional temperature field. Infrared thermal imaging provides visual and diversified information of body temperature distribution marked with different colors to the clinicians. It provided an important way for disease diagnosis by analyzing the heat map and the difference of surface temperature [17]. In many diseases the blood flow varied and influenced the skin temperature. IR imaging offers a useful and noninvasive approach to disease diagnosis and treatment (as therapeutic aids), in particular in the areas of rheumatology, dermatology, orthopaedics, and circulatory abnormalities [18]. With the development of infrared thermal imaging technology, it was used not only in breast cancer screening and diagnosis [19], but also in the diagnosis of a variety of diseases, for example, for early diagnosis and study of variety of tumors [20-24], the diagnosis of breast disease [25-27], the study of peritoneal inflammatory diseases [28], early screening of myocardial ischemia [29], the study of the clinical efficacy of herbs and testing Chinese herbal nature [30, 31], the research of back acupoints thermal distribution on bronchial asthma patients [32], the thermographic observation of shoulder-hand syndrome following stroke [33], the study of the infrared radiant track along lung meridian of patients with lung diseases [34], the study of warm needling used in the treatment of lumbar disc herniation [35], and the study of early diagnosis of thyroid disease $[36,37]$. It was reported that the far infrared thermogram was applied as an index for diagnosis of acute peripheral facial paralysis [38].

Zheng classification study based on infrared thermal imaging technology has not been reported before. Our previous studies showed that under physiological conditions, the relative temperature of viscera and bowels was regulated by a special rule and the relative temperature in descending order was ordered as follows: lung $>$ descending colon $>$ ascending colon $>$ liver $>$ stomach $>$ kidney $>$ small intestine [6]. In this trial, the relative temperature of viscera and bowels of patients with cold-phlegm obstructing lung or phlegmheat obstructing lung was different from that of normal group, although the relative temperature of lung was highest, followed by lower temperature of descending colon and ascending colon. Compared with normal group, the relative temperature in the region of lung, descending colon, ascending colon, small intestine, and stomach was significantly increased in phlegm-heat obstructing lung syndrome group. While compared with normal group, the relative temperature in lung region and descending colon region was significantly increased in cold-phlegm obstructing lung syndrome group, but the relative temperature in the region of ascending colon, small intestine, and stomach was not significantly different. In the comparison between different Zheng classification, the relative temperature of lung and small intestine significantly increased in phlegm-heat obstructing lung syndrome group than in cold-phlegm obstructing lung syndrome group, and the difference was significant. Most of the viscera and bowels relative temperature in phlegm-heat obstructing lung syndrome group was higher than that in cold-phlegm obstructing lung syndrome group. The result indicated that phlegm-heat obstructing lung syndrome influenced viscera and bowels function deeper than cold-phlegm obstructing lung syndrome.

Pairing of the viscera and bowels is an important theory, which provides guidance to traditional Chinese medicine (TCM) clinical practice. The investigation has been the focus of research on the basic theory of TCM [39]. The results showed that the organs function was influenced to some degree when the physiological function of the lung is abnormal. From the point of view of infrared thermal imaging technology, the relative temperature of organs in different Zheng classification was not in the same level. But both in phlegm-heat obstructing lung syndrome group and in cold-phlegm obstructing lung syndrome group, the relative temperature of lung and descending colon was consistently influenced. It provided clinical evidence to the theory that the lung and large intestine are exteriorly-interiorly related. The application of infrared thermal imaging technology provided objective method for medical diagnosis and treatment in the field of Zheng studies and provided a new methodology for Zheng classification.

In the clinic, the traditional Chinese medicine Zheng classification of pulmonary diseases presented diversity. Experienced Chinese medicine practitioners usually make syndrome differentiation by four examinations obtained. But some Zheng classification diagnosed by some doctors were still not clear; they directly reduced the efficacy of treatment and even aggravated the patient's illness. In the clinic, Zheng classification diagnostic tools were lacking at present. The infrared thermal images characteristics of different Zheng classifications of pulmonary disease were distinctly different. The influence on viscera and bowels was deeper in phlegmheat obstructing lung syndrome group than in cold-phlegm 
obstructing lung syndrome group. The viscera and bowels relative temperature of patients with different Zheng classification of pulmonary diseases was different. The trial indicated that it is helpful to diagnose Zheng classification and to improve the diagnosis rate by analyzing the infrared thermal images of patients.

\section{Conclusion}

The infrared thermal images characteristics of different Zheng classifications of pulmonary disease were distinctly different. The influence on viscera and bowels was deeper in phlegm-heat obstructing lung syndrome group than in coldphlegm obstructing lung syndrome group. It is helpful to diagnose Zheng classification and to improve the diagnosis rate by analyzing the infrared thermal images of patients. The application of infrared thermal imaging technology provided objective measure for medical diagnosis and treatment in the field of Zheng studies and provided a new methodology for Zheng classification.

\section{Conflict of Interests}

The authors do not have a direct financial relation with the commercial identity mentioned in the paper that might lead to a conflict of interests for any of the authors.

\section{Acknowledgments}

The trial was supported by National Key Basic Research and Development Program "The research of literature review and comprehensive analysis on the theory of lung and large intestine are exterior-interior related" (no. 2009CB522701). The authors acknowledge the assistance of all the members of the project. They thank FENG Cui-ling, TIAN Shu-ying, all from Dongzhimen Hospital Affiliated to Beijing University of Chinese Medicine for providing cases to the clinical trial. We thank Kang Lei and LIU Yu-chao for recruiting patients.

\section{References}

[1] E. Y. K. Ng, "Is thermal scanner losing its bite in mass screening of fever due to SARS?” Medical Physics, vol. 32, no. 1, pp. 93-97, 2005.

[2] U. R. Acharya, E. Y. Ng, J. H. Tan et al., "Thermography based breast cancer detection using texture features and Support Vector Machine," Journal of Medical Systems, vol. 36, no. 3, pp. 1503-1510, 2012.

[3] W. K. Ng, Y. K. Ng, and Y. K. Tan, "Qualitative study of sexual functioning in couples with erectile dysfunction prospective evaluation of the thermography diagnostic system," Journal of Reproductive Medicine for the Obstetrician and Gynecologist, vol. 54, no. 11-12, pp. 698-705, 2009.

[4] J. H. Tan, E. Y. K. Ng, and U. R. Acharya, "Evaluation of tear evaporation from ocular surface by functional infrared thermography," Medical Physics, vol. 37, no. 11, pp. 6022-6034, 2010.

[5] C. Purslow and J. S. Wolffsohn, "Ocular surface temperature: a review," Eye and Contact Lens, vol. 31, no. 3, pp. 117-123, 2005.
[6] J. X. Ni, S. H. Gao, T. Tian et al., "Visualized study on the viscera and bowels function of healthy people based on the infrared thermal imaging technology," Journal of Beijing University of Traditional Chinese Medicine, vol. 10, no. 35, p. 666, 2012.

[7] Chronic Obstructive Pulmonary Disease Study Group of Chinese Society of Respiratory Diseases, "Chronic obstructive pulmonary disease treatment guidelines," Chinese Journal of Tuberculosis and Respiratory Diseases, vol. 30, no. 1, pp. 8-17, 2007.

[8] Asthma Study Group of Chinese Society of Respiratory Diseases Bronchial, "Asthma prevention and treatment guidelines," Chinese Journal of Tuberculosis and Respiratory Diseases, vol. 26, no. 3, pp. 132-138, 2003.

[9] National Clinical Professional Meeting of Chronic Bronchitis, "Chronic bronchitis clinical diagnosis and the efficacy criteria," Journal of Chengdu University of Traditional Chinese Medicine, vol. 17, no. 3, pp. 52-53, 1994.

[10] T. C. M. Zheng, Diagnosis and Efficacy Standard, Nanjing University Press, Nanjing, China, 1994.

[11] National Science and Technology Approval Committee, TCM Terms, Science Press, Beijing, China, 2004.

[12] A. P. Lu and K. J. Chen, "Pondering on syndrome differentiation of disease," Chinese Journal of Integrated Traditional and Western Medicine, vol. 25, no. 9, pp. 843-844, 2005.

[13] A. P. Lu and S. Li, "Principle and application of Chinses medicine syndrome differentiation and disease classification," Chinese Journal of Integrated Traditional and Western Medicine, vol. 30, no. 1, article 84, 2010.

[14] J. Y. Yao, S. J. Miao, X. M. Ji et al., "Analysis of death of main diseases among urban populations in China," Chinese Medical Practice, vol. 5, no. 10, article 15, 2010.

[15] C. D. Mathers and D. Loncar, "Projections of global mortality and burden of disease from 2002 to 2030," PLoS Medicine, vol. 3, no. 11, pp. 2011-2030, 2006.

[16] Z. B. Zhang and Y. Y. Wang, "Review and hypothesis put forward of the name and classification of Zheng," Journal of Beijing University of TCM, vol. 26, no. 2, article 1, 2003.

[17] A. Bao and Q. L. Hao, "Infrared imaging applications in the field of medical diagnostics," in Proceedings of the Preventive Treatment of Disease and Sub-Health Forum and the First SubHealth Meridian Conditioning Symposium, pp. 34-39, Beijing, China.

[18] L. J. Jiang, E. Y. Ng, A. C. Yeo et al., "A perspective on medical infrared imaging," Journal of Medical Engineering \& Technology, vol. 29 , no. 6 , pp. $257-267$.

[19] J. F. Head, F. Wang, C. A. Lipari et al., "The important role of infrared imaging in breast cancer," IEEE Engineering in Medicine and Biology Magazine, vol. 19, no. 3, pp. 52-57, 2000.

[20] J. H. Yang, H. L. Zhang, Y. X. Kang et al., "Application of infrared imaging technology for the diagnosis of malignant tumors," Modern Oncology, vol. 17, no. 9, pp. 1817-1819, 2009.

[21] J. X. Chen, K. H. Miao, and L. Li, "The clinical value of diagnosis of adrenal tumors by medical infrared thermal imaging technology," China Journal of Modern Medcine, vol. 21, no. 6, pp. 650-651, 2005.

[22] S. W. Lu, G. Q. Zhang, H. Li et al., "Inspection on hepatocellular tumorigenesis and metastasis of experimental nude mice by Infrared Imaging System," Laser \& Infrared, vol. 36, no. 7, pp. 573-576, 2006.

[23] G. S. Lu, W. Pang, and L. L. Wang, "The application of the diagnosis of nasopharyngeal cancer by infrared imaging system," Laser \& Infrared, vol. 31, no. 1, pp. 42-44, 2001. 
[24] M. Y. Xia, Y. J. Zhou, J. Li et al., "The value of the far infrared thermal scan imaging system for early diagnosis of colon cancer," Chinese Journal of Medical Imaging Technology, vol. 12, no. 2, article 153, 2004.

[25] Y. Yuan, Q. Wang, R. Li et al., “Thermal texture maps in differential diagnosisof breast tumors," Chinese Journal of Medical Imaging Technology, vol. 20, no. 12, pp. 1803-1805, 2004.

[26] H. Yang, Q. Y. Lin, A. Xu et al., "Infrared thermal imaging technology and its preliminary study on breast disease assessment," Life Science Instruments, vol. 5, pp. 39-43, 2007.

[27] Y. H. Liu, R. Li, and X. M. Wang, "The diagnostic value of infrared thermography for various breast diseases," Journal of Clinical and Experimental Medicine, vol. 6, no. 1, pp. 34-36, 2007.

[28] Z. H. Zhang, "The characteristics of IR Thermo-image for the intra-abdominal inflammatory diseases and tumour," Laser \& Infrared, vol. 26, no. 2, pp. 149-151, 1996.

[29] H. He, F. Z. Zhao, N. Li et al., "The analysis of influencing factors to 120 cases myocardial ischemia changed by thermal tomography," Clinical Journal of Medical Officers, vol. 38, no. 1, pp. 148-149, 2010.

[30] X. M. Li, X. Q. Huang, X. Li et al., “Thermal effects of cold \& cool Traditional Chinese Medicine of channel tropism of stomach on stomach-heat syndrome rats using thermal texture maps system," Acta Laser Biology Sinica, vol. 16, no. 2, pp. 179-185, 2007.

[31] M. Zhu, Y. H. Li, T. X. Lin et al., "Application of TTM system for testing Chinese herbal nature," Chinese Journal of Stereology and Mage Analysis, vol. 12, no. 1, pp. 53-58, 2007.

[32] S. H. Ma and R. X. Chen, "Clinical studies on back thermal acupoints distribution in bronchial asthma (remission) patients," Jiangxi Journal of Traditional Chinese Medicine, vol. 42, no. 337, pp. 30-32, 2011.

[33] X. P. Yun, H. Z. Guo, and W. Y. Li, "The thermographic observation of shoulder-hand syndrome following stroke," Chinese Journal of Physical Therapy, vol. 24, no. 5, pp. 280-281, 2001.

[34] J. S. Xu, X. L. Hu, and G. Y. Yang, "The observation of the infrared radiant track along lung meridian of patients with lung diseases," Journal of Fu Jian College of TCM, vol. 15, no. 6, pp. 18-20, 2005.

[35] Y. Zhang, J. Shi, F. A. Li et al., "The study of TTM on the treatment of kidney lumbar disc herniation with abdominal temperature needle," Clinical Journal of Chinese Medicine, vol. 3, no. 9, pp. 57-59, 2011.

[36] T. Yang, "The diagnosis of TTM in tubercle thyroid disases (8 cases report)," Chinese Imaging Journal of Integrated Traditional and Western Medicine, vol. 6, no. 2, pp. 107-110, 2008.

[37] X. M. Li, Y. Wang, and X. M. Che, "The contrastive analysis of Thyroid disease by TTM, (infrared thermal fault) and color Doppler," The Journal of Medical Theory and Practice, vol. 24, no. 4, article 445, 2011.

[38] Y. H. Gao, Y. L. Xue, L. Yun et al., "Changes of cephalofacial far infrared thermogram as an evaluating index for treatment of acute peripheral facial paralysis," Journal of Clinical Rehabilitative Tissue Engineering Research, vol. 14, no. 13, pp. 2373-2376, 2010.

[39] J. X. Ni and S. H. Gao, "Understanding the viscera-related theory that the lung and large intestine are exterior-interiorly related," Journal of Traditional Chinese Medicine, vol. 32, no. 2, article 293, 2012. 


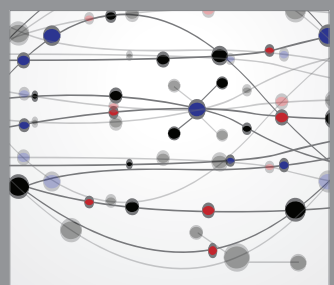

The Scientific World Journal
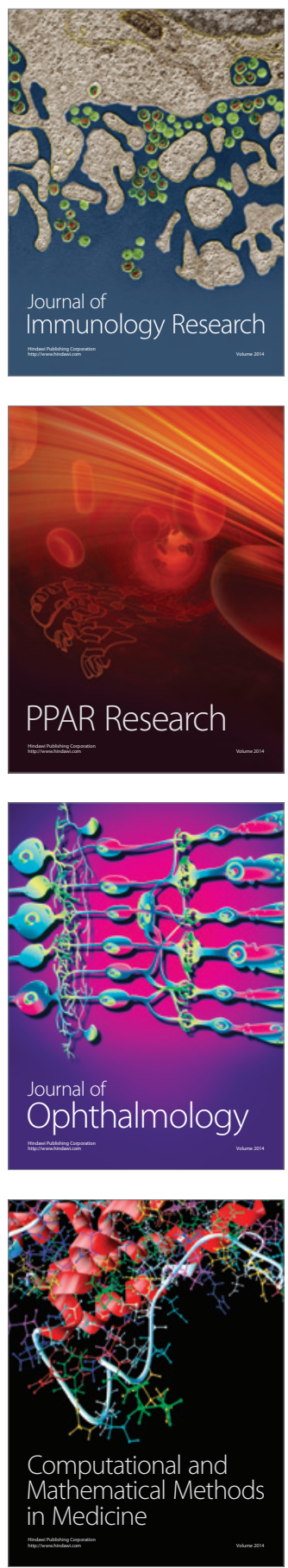

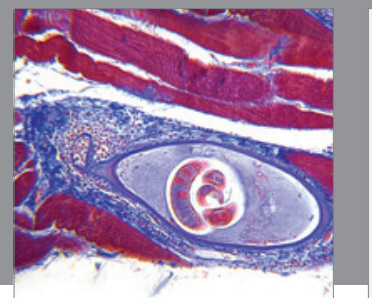

Gastroenterology

Research and Practice
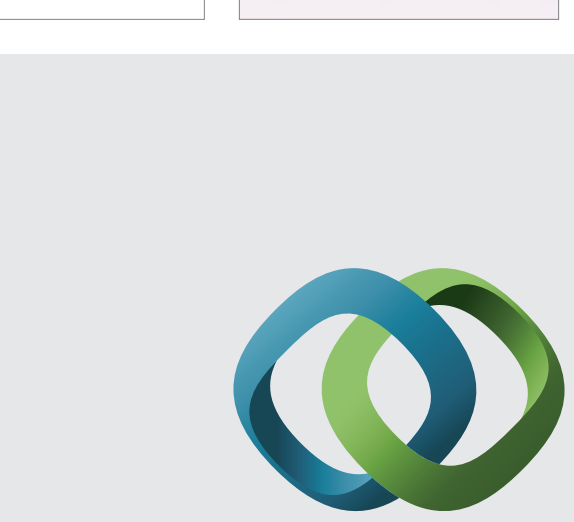

\section{Hindawi}

Submit your manuscripts at

http://www.hindawi.com
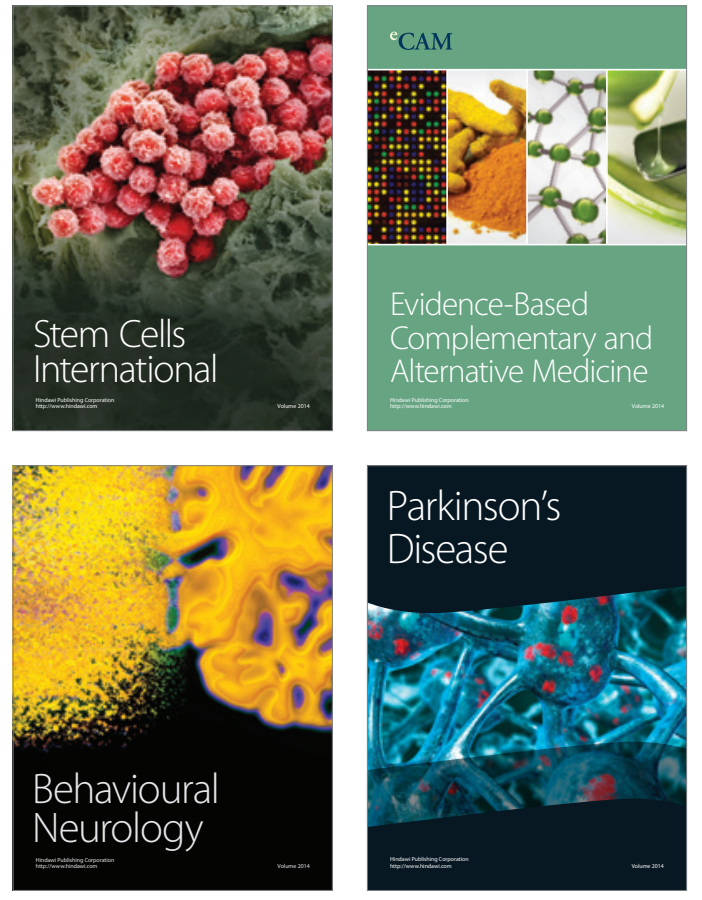
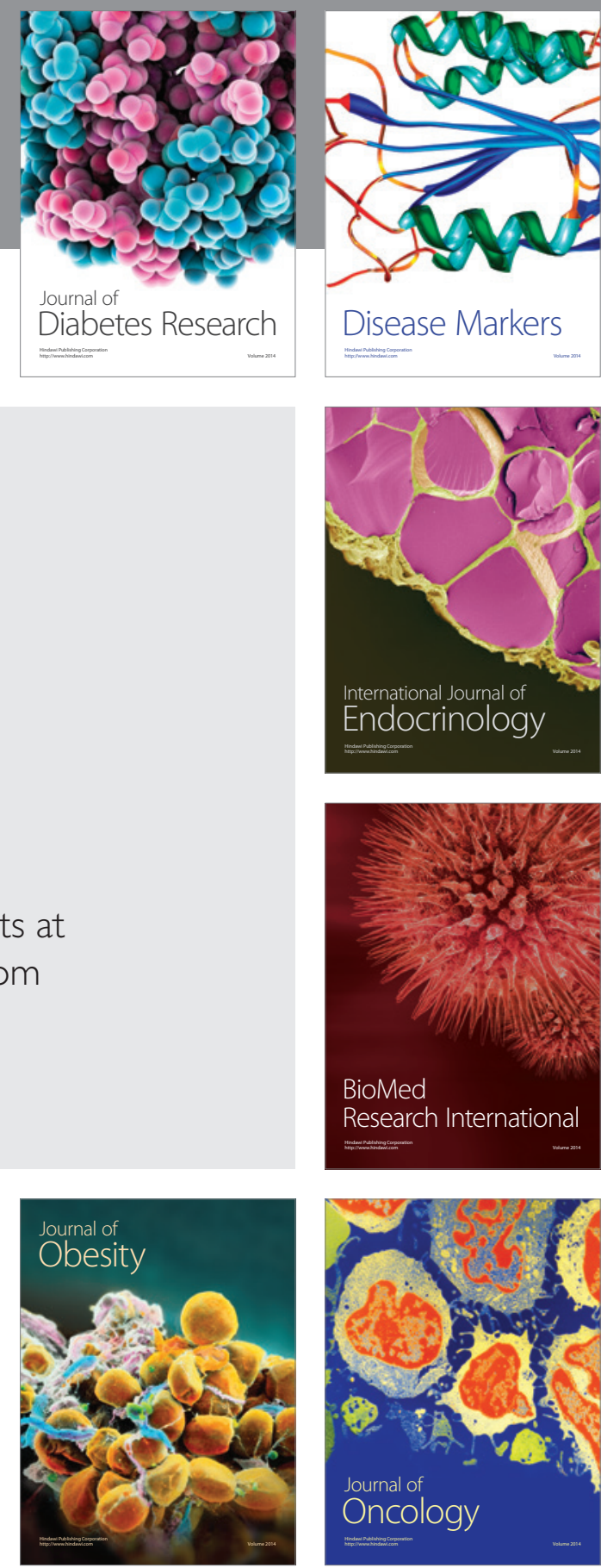

Disease Markers
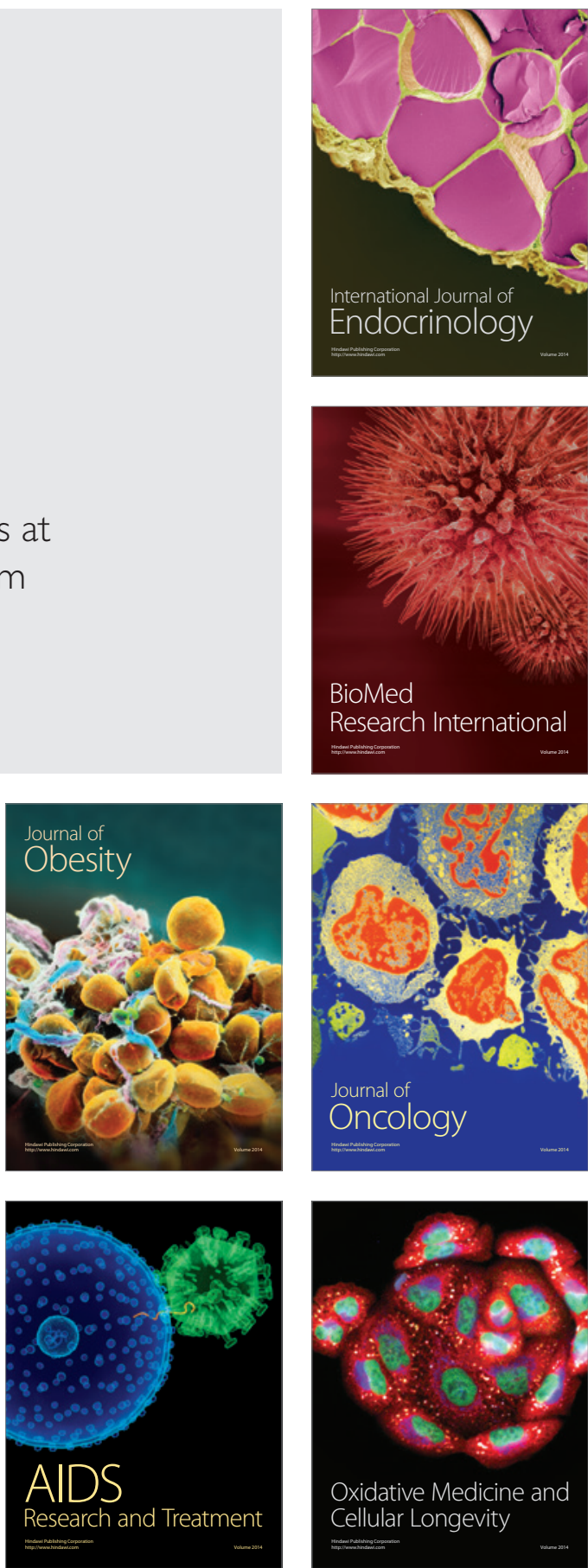